\title{
Mycobacterium xenopi pulmonary infection in an HIV infected patient under highly active antiretroviral treatment
}

\author{
C Bachmeyer, L Blum, S Stelianides, B Benchaa, N Gruat, O Danne
}

Département de Médecine Interne, Hôpital Laënnec, F-60109 Creil, France C Bachmeyer

Médecine Générale, Hôpital René Dubos, Pontoise, France

L Blum

B Benchaa

O Danne

Pneumologie, Centre Hospitalier du Vexin, Magny en Vexin,

France

S Stelianides

N Gruat

Correspondence to: Dr C Bachmeyer claude.bachmeyer@ch-creil.fr

Received 28 February 2001 Returned to authors 30 June 2001

Revised version received 13 July 2001

Accepted for publication

1 August 2001

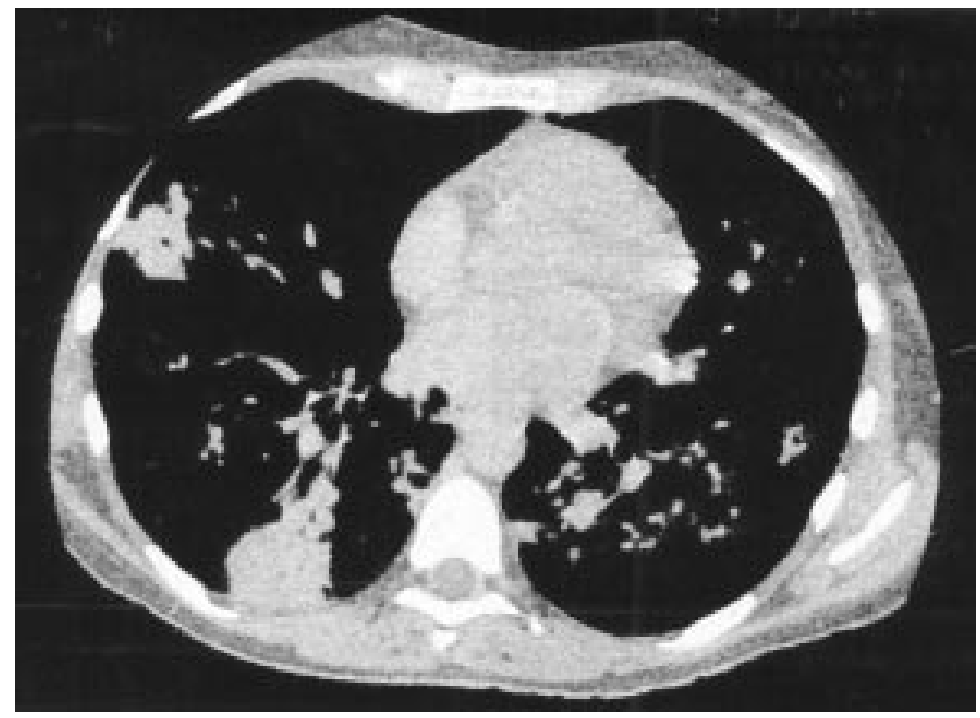

Figure 1 CT scan of the chest showing multiple nodular lesions and cavitation of the left lower lobe.

\begin{abstract}
Highly active antiretroviral therapy (HAART) is responsible for a striking reduction in AIDS related morbidity and mortality by partly restoring immune function. However, HAART can also precipitate the development of clinically apparent opportunistic infections in patients with latent infections. We report a case of an HIV infected patient who developed granulomatous nodular and cavitatory lesions of the lungs due to Mycobacterium xenopi as a manifestation of the immune restoration syndrome.

(Thorax 2001;56:978-979)
\end{abstract}

Keywords: Mycobacterium xenopi; HIV; HAART; immune restoration syndrome

Highly active antiretroviral therapy (HAART) is responsible for a striking reduction in AIDS related morbidity and mortality by partly restoring immune function. ${ }^{1}$ Improvement and resolution of opportunistic infections has been most commonly observed. Unfortunately, HAART can also precipitate the development of clinically apparent opportunistic infections in patients with latent infections within the first weeks of treatment. ${ }^{1-4}$ We report a Mycobacterium xenopi pulmonary infection presenting with granulomatous nodular and cavitatory lesions as a complication of the immune restoration syndrome.

\section{Case report}

In July 1999 treatment with zidovudine, lamivudine, and fortovase associated with cotrimoxazole was started in a 35 year old woman with HIV-1 infection in whom the CD4+ lymphocyte count was $48 / \mathrm{mm}^{3}$ and the viral load was 150229 HIV RNA copies/ml. One month later her temperature was $38^{\circ} \mathrm{C}$ and isolated serum positivity for cryptococcal antigen was discovered which was controlled with fluconazole. In September 1999 she presented with cough and a fever of $38.5^{\circ} \mathrm{C}$. A chest radiograph and CT scan demonstrated bilateral nodules of the lungs and cavitations in the left lower lobe, but no lymph nodes (fig 1). At this time the CD4+ lymphocyte count was $225 / \mathrm{mm}^{3}$ and the viral load was 114 HIV RNA copies $/ \mathrm{ml}$. The tuberculin skin reaction was negative and liver function tests were unremarkable. Serological tests for Coxiella burnetii, Mycoplasma pneumoniae, Chlamydia pneumoniae, and Legionella were either negative or did not suggest recent infection. Direct microscopic examination of sputum was negative. Bronchoscopic examination, direct search for pathogens on bronchoalveolar lavage specimens and transbronchial biopsy specimen were normal or negative. A CT guided percutaneous biopsy of a nodule was therefore performed. Histological examination showed an epithelioid cell granuloma. As mycobacterial infection was suspected, efavirenz was substituted for fortovase, and antimycobacterial treatment combining isoniazid, rifampicin, and ethambutol was commenced. Cultures from one sputum out of three grew Mycobacterium xenopi which was sensitive to all of the drugs, whereas cultures from the transbronchial biopsy specimen were negative. A dramatic improvement in clinical symptoms and in radiographic features was observed within 2 months.

\section{Discussion}

The development of opportunistic infections during the first 2 months of HAART has 
recently been described, including cytomegalovirus, Cryptococcus neoformans, and Mycobacterium avium intracellulare. ${ }^{1-4}$ Indeed, clinically silent infections may become apparent as a result of restoration of specific immune activity against microbial pathogens. This results from intensified inflammatory responses caused by the sudden increase in lymphocytes specifically reacting, for example, against mycobacterial antigens in mycobacterial infections. ${ }^{4}$

We present a case of $M$ xenopi pulmonary infection as a complication of the immune restoration syndrome. To the best of our knowledge only one similar case has been reported, who presented with nodular lung lesions and left sided pleurisy. ${ }^{4}$ Mycobacterium xenopi is a scotochromogenic, acid fast, slow growing, non-tuberculous bacillus, growing optimally at $42^{\circ} \mathrm{C}$. It is usually considered as a commensal, saprophyte, or environmental contaminant. Its pathogenicity is relatively low and infection requires impairment of host immunity. Infection usually occurs in the lungs in patients with chronic obstructive pulmonary disease, chronic alcoholism, diabetes mellitus, and malignancies. ${ }^{5}{ }^{6}$ Radiographically it presents with multinodular densities and cavitations. Granuloma formation is typical of such an infection when available. No treatment guidelines exist for pulmonary $M$ xenopi infection, but a combination of three or four drugs which might include streptomycin, ethionamide, and ethambutol or rifampicin continued for 18-24 months seems reasonable. ${ }^{5}$ In vitro drug susceptibility and response to chemotherapy are unpredictable. In a few cases surgical resection resulted in cure when antibiotics were ineffective. Of note, diagnosis of uncommonthat is, other than $M$ avium complex and $M$ kansasii-non-tuberculous mycobacterial disease in HIV negative patients relies on the criteria of the American Thoracic Society: (a) either repeated isolation from a non-sterile site or a single isolation of $M$ xenopi from a normally sterile localisation and (b) either the presence of one or more symptoms indicative of pulmonary disease or an abnormal chest radiograph, in the absence of other pathogens or illnesses. ${ }^{8}$
Mycobacterium xenopi has been reported as being responsible for pulmonary and extrapulmonary infections in HIV infected patients. $^{5-7}$ Fever and cough are common symptoms in these patients, and fever is significantly more frequent in patients with AIDS than in non-AIDS patients. Radiographic findings include interstitial and mixed disease, rarely a reticulonodular pattern and cavitary disease, but adenopathy is unusual. Most patients have advanced HIV infection with a CD4 cell count of $<100 / \mathrm{mm}^{3}$. Differential diagnosis includes Pneumocystis carinii pneumonia, other mycobacterial infections, ${ }^{7}$ and sarcoidosis which has also recently been described as the result of the immune restoration syndrome. ${ }^{9}$

In our patient the diagnosis of $M$ xenopi pulmonary infection was likely on clinical, radiological, and histological findings, despite only one specimen of sputum being positive for the organism. Indeed, the diagnostic criteria of the American Thoracic Society were not fulfilled. However, we consider that this result should not be attributed to environmental contamination.

1 Sepkowitz KA. Effect of HAART on natural history of AIDS-related 1998;351:228-30

2 Michelet C, Arvieux C, Francois C, et al. Opportunistic infections occurring during highly active antiretroviral infections occurring during highly

3 Race EM, Adelson-Mitty J, Kriegel GR, et al. Focal mycobacterial lymphadenitis following initiation of protease-inhibitor therapy in patients with advanced HIV-1 nfection. Lancet 1998;351:252-5.

4 Foudraine NA, Hovenkamp E, Notermans DW, et al. Immunopathology as a result of highly active antiretroviral therapy in HIV-1-infected patients. AIDS 1999;13:177-84.

5 Jiva TM, Jacoby HM, Weymouth LA, et al. Mycobacterium xenopi: innocent bystander or emerging pathogen? Clin Infect Dis 1997;24:226-32.

6 El-Solh AA, Nopper J, Abdul-Khoudoud MR, et al. Clinical and radiographic manifestations of uncommon pulmonary nontuberculous mycobacterial disease in AIDS patients. Chest 1998;114:138-45.

7 Juffermans NP, Verbon A, Danner SA, et al. Mycobacterium xenopi in HIV-infected patients: an emerging pathogen. AIDS 1998;12:1661-6.

8 American Thoracic Society. Diagnosis and treatment of disease caused by nontuberculous mycobacteria. Am Rev Respir Dis 1990;142:940-53.

9 Naccache JM, Antoine M, Wislez M, et al. Sarcoid-like pulmonary disorder in human immunodeficiency virusRespir Crit Care Med 1999;159:2009-13. 